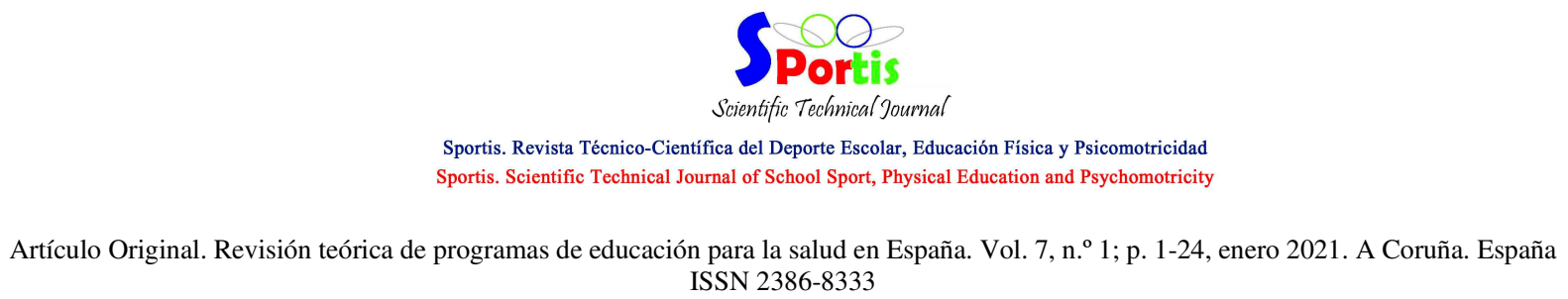
ISSN 2386-8333

\title{
Revisión teórica de programas de educación para la salud en España
}

\section{Theoretical review of health education programs in Spain}

\author{
Federico Salvador Pérez ${ }^{1}$, José Joaquín Muros Molina ${ }^{1}$, Vanesa María Gámiz Sánchez ${ }^{1}$, \\ Félix Zurita Ortega ${ }^{1}$ \\ ${ }^{1}$ Facultad de Ciencias de la Educación. Campus de Cartuja. Universidad de Granada
}

Contacto: federicosalvador@gmail.com

Cronograma editorial: Artículo recibido: 13/05/2020 Aceptado: 8/10/2020 Publicado: 01/01/2021

https://doi.org/10.17979/sportis.2021.7.1.6530

\section{Resumen}

Los programas de intervención orientados hacia hábitos de vida saludables son cada vez más imprescindibles en la sociedad actual, siendo las poblaciones jóvenes los principales interesados en los mismos, por tanto, se presenta un estudio de revisión de literatura, con los objetivos de realizar una revisión teórica de artículos relacionados con programas de salud y localizar y analizar un programa de salud en cada una de las provincias de España. Los criterios de inclusión fueron que se tratase de estudios realizados en España, que fuesen de los 10 últimos años, que se hubiese desarrollado un programa de intervención y que se encontrasen en revistas indexadas en SCOPUS o Web of Science. Como resultados principales se debe señalar que los programas de salud se hallan reflejados en todas las provincias de España y en todos los casos se ha detectado al menos un estudio en los últimos diez años. Se puede concluir señalando como en todas las provincias de España hay un interés por determinar aspectos relacionados con la salud.

\section{Palabras Clave}

Programa de Salud; Nutrición; Hábitos saludables. 


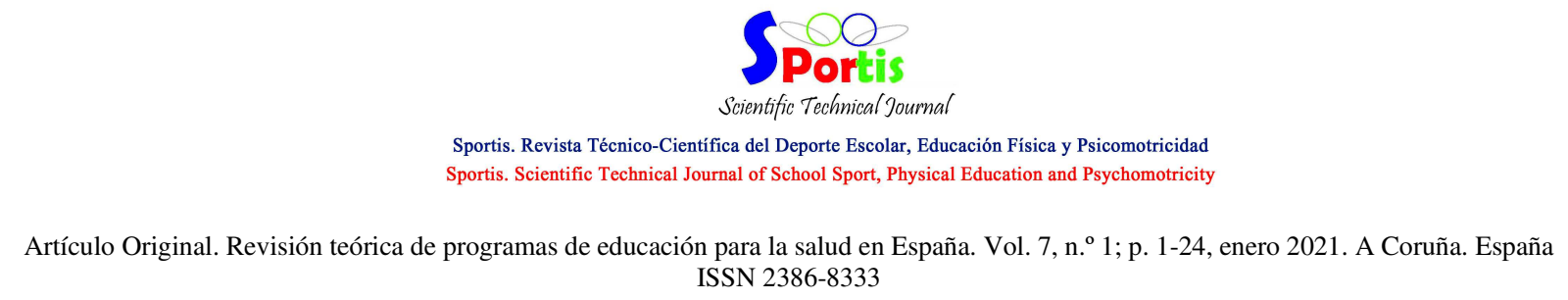

\section{Abstract}

Intervention programs oriented towards healthy lifestyle habits are increasingly essential in today's society, with young populations being the main interested in them, therefore, a literature review study is presented, with the aim of conducting a review theoretical articles related to health programs and to locate and analyze a health program in each of the provinces of Spain. The inclusion criteria were that they were studies carried out in Spain, that they were from the last 10 years, that an intervention program had been developed and that they were in journals indexed in SCOPUS or WEB of Science. As main results, it should be noted that health programs are reflected in all provinces of Spain and in all cases at least one study has been detected in the last ten years. It can be concluded by pointing out how in all the provinces of Spain there is an interest in determining aspects related to health.

\section{KeyWords}

Health Program; Nutrition; Healthy habits.

\section{Introducción}

De las enfermedades crónicas más comunes, la obesidad forma parte de un problema muy generalizado en la población. Su estudio y prevención forman parte de una gran preocupación en la comunidad científica. Además, la gran repercusión en la salud de las personas, hace que se intente tratar desde los primeros años de vida. Cabe resaltar, que una de las problemáticas y preocupaciones extendidas en el mundo en relación al sobrepeso son las cifras crecientes de obesidad infantil (Bacardí-Gascón, Jiménez-Cruz, Jones, y Guzmán, 2007). Los cálculos aproximados señalan que unos 250 millones de personas tienen obesidad en todo el mundo, enfatizando que con sobrepeso estarían el doble o el triple (Speiser, Rudolf y Anhalt, 2005).

Se puede entender la obesidad como término en cuanto a una condición compleja de muchos factores al que le acompañan un exceso elevado de grasa corporal (Poletti, Oscar y Lilian, 2003) incluyendo componentes genéticos (Rey, Bresson y Abadie, 1994) y ambientales (Gortmaker, Must, Sobol y Pterson, 1996). Por tanto, constituyen uno de los principales problemas de salud en todo el mundo y representan un desafío continuo tanto para los países en desarrollo como para los desarrollados. Las cifras proporcionadas por la Organización Mundial de la Salud (en adelante OMS) así lo demuestran, ya que la prevalencia de obesidad aumentó desde 1980 a 2008 un 5\% en hombres y un 6\% en mujeres.

Para citar este artículo utilice la siguiente referencia: Salvador, F.; Muros, J.J.; Gámiz, V.Ma.; Zurita, F. (2021). Revisión teórica de programas de educación para la salud en España. Sportis Sci J, 7 (1), 1-24. https://doi.org/10.17979/sportis.2021.7.1.6530 


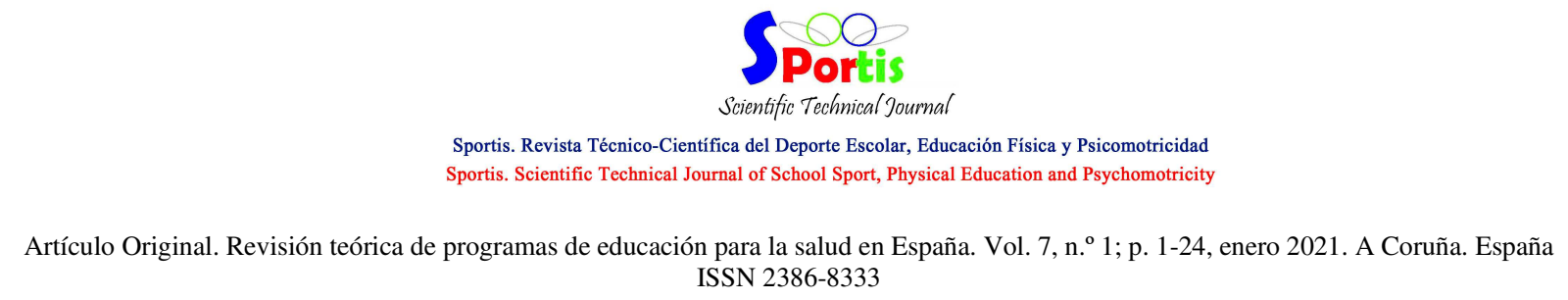

Además, la OMS estima que cada año fallecen 2,8 millones de personas por sobrepeso u obesidad.

La obesidad durante la primera etapa de la vida tiene importantes consecuencias a corto y largo plazo. En el primer caso manifiesta consecuencias desfavorables en cuanto a la presión arterial, los lípidos, el metabolismo de los carbohidratos, la autoestima y la calidad de vida (Gidding, Bao, Srinivasan y Berenson, 1995; Urrutia-Rojas et al., 2006).

A largo plazo de obesidad en la etapa adulta se presenta un mayor riesgo de hipertensión arterial, diabetes, enfermedades cardiovasculares, etc. (Dietz, 1998; Serdula, Ivery, Coates, Freedman, Williamson y Byers, 1993).

En la etapa infantil, si se almacena grasa corporal en exceso, sobre todo de tipo androide (acumulada en el tronco y el abdomen) puede mantenerse en el periodo adolescente y manifestar ya en el período adulto efectos fisiológicos y patológicos de morbilidad y mortalidad (Dietz, 1998; Nieto, Szclo y Comstock, 1992). También se asocia a problemas psiquiátricos tan graves como la depresión (Erickson, Robinson, Haydel y Killen, 2000) carencia de autoestima (French, Story y Perry, 1995) y la transformación de la percepción de la imagen corporal (Sullivan et al, 1993).

Según diferentes estudios, en 2010 cuarenta millones de niños menores de cinco años tenían sobrepeso en el mundo. La obesidad infantil está íntimamente relacionada con la obesidad en la etapa adulta, además de muerte prematura o discapacidad. En muchas ocasiones los niños que presentan este problema coincide que tienen problemas de respiración, hipertensión arterial, mayor riesgo de fracturas, resistencia a la insulina e incluso efectos psicológicos (Petry, Barry, Pietrzak y Wagner, 2008; Tobisch, Blatniczky y Barkai, 2015).

Los hábitos alimenticios y las predilecciones por unos alimentos u otros están sufriendo diversos cambios con el ritmo de la sociedad actual invirtiendo menos tiempo al día para adquirir alimentos o preparar las diferentes comidas diarias. Esto hace que las personas, al carecer de tiempo como antes, busquen en la comida rápida y en los alimentos sencillos en su preparación una forma de salir del paso. Lo normal es que este tipo de alimentos sean de origen animal (carnes y derivados) o con azúcares refinados (lo que conlleva un aumento de grasas saturadas y colesterol en la dieta) (Aranceta, 2001; Durá, 2001; Moreno, Sarría y Para citar este artículo utilice la siguiente referencia: Salvador, F.; Muros, J.J.; Gámiz, V.Mª .; Zurita, F. (2021). Revisión teórica de programas de educación para la salud en España. Sportis Sci J, 7 (1), 1-24. https://doi.org/10.17979/sportis.2021.7.1.6530 http://revistas.udc.es/ 


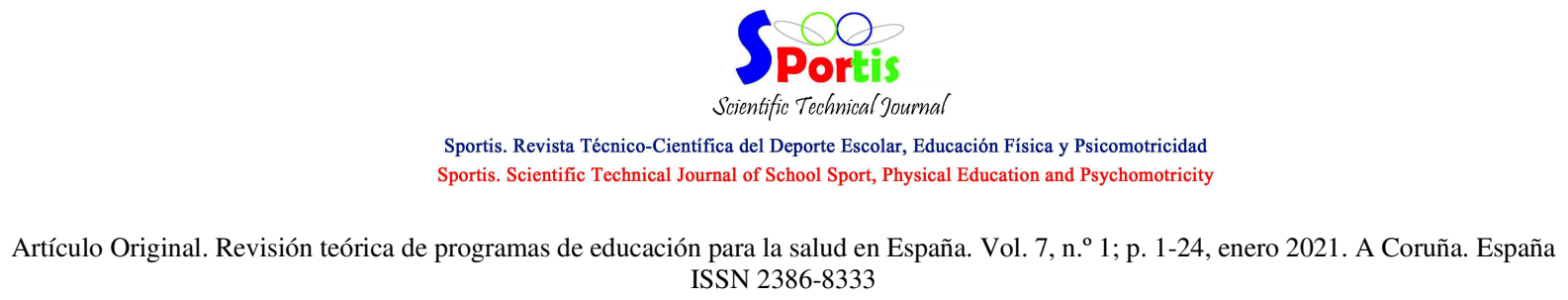

Popkin, 2002; Rodríguez, Banegas, Graciani, Hernández y Rey, 1996; Sánchez-Villegas, Martínez, De Irala y Martínez-González, 2002; Tur et. al, 2005).

Los escolares que precisamente son los que más necesitan de una dieta saludable para su correcto desarrollo son los que más influenciados están por lo anterior. Esto hace que se deba de tener una especial atención con este grupo (Durá, 2001; Iturbe, Emparanza y Perales, 1999; Samuelson, Bratteby, Enghardt y Hedgren, 2000; Serra-Majem, García-Closas, Ribas, Pérez-Rodrigo y Aranceta, 2001; Tur, Romaguera y Pons, 2004).

Realizar actividad física en edades tempranas da lugar a obtener beneficios directos de mejoras biológicas reflejadas en los niveles de grasa corporal o desarrollo óseo e incluso en el incremento del desarrollo cardiovascular. También se mejoran factores psicológicos tan importantes como la autoestima y el bienestar o en factores sociales como aspectos morales o sociales. El realizar actividad física en la etapa escolar reporta beneficios que contribuirán a seguir siéndolos una vez que concluya la etapa escolar obligatoria (Devís y Garde, 2002).

La promoción de la salud persigue que las personas de manera individual y colectiva consigan obtener una superior calidad de vida, sin olvidar que son entidades biológicas y sociales que demandan ser tratadas por los profesionales sanitarios de manera conjunta con el objetivo de alcanzar ser comunidades saludables (Casas y Klijn, 2006).

Para el desarrollo y puesta en práctica de los proyectos de promoción de la salud se pueden seguir varios elementos interconectados que podrían ser adecuados. Estos elementos propuestos son: la creación de estrategias públicas y entornos saludables, consolidar la participación de la ciudadanía, orientar correctamente los servicios de salud y potenciar las habilidades de cada persona con el objetivo de promover la salud (Moysés, Moysés y Krempel, 2004).

Para los proyectos de promoción de vida saludable se debería de tener en cuenta tanto lo anterior como lo que otros estudios aportan ya que apoyan la garantía de apostar por esta clase de motivación (Moreno, Cervelló y Borges, 2010).

Arriscado, Muros, Zabala y Dalmau (2014) concluyen que invertir para el fomento de estilos de vida saludables es el camino adecuado para ahorrar tratamientos de enfermedades y patologías que provengan de la falta de actividad física y de un mal planteamiento dietético.

Para citar este artículo utilice la siguiente referencia: Salvador, F.; Muros, J.J.; Gámiz, V.Ma.; Zurita, F. (2021). Revisión teórica de programas de educación para la salud en España. Sportis Sci J, 7 (1), 1-24. https://doi.org/10.17979/sportis.2021.7.1.6530 http://revistas.udc.es/ 


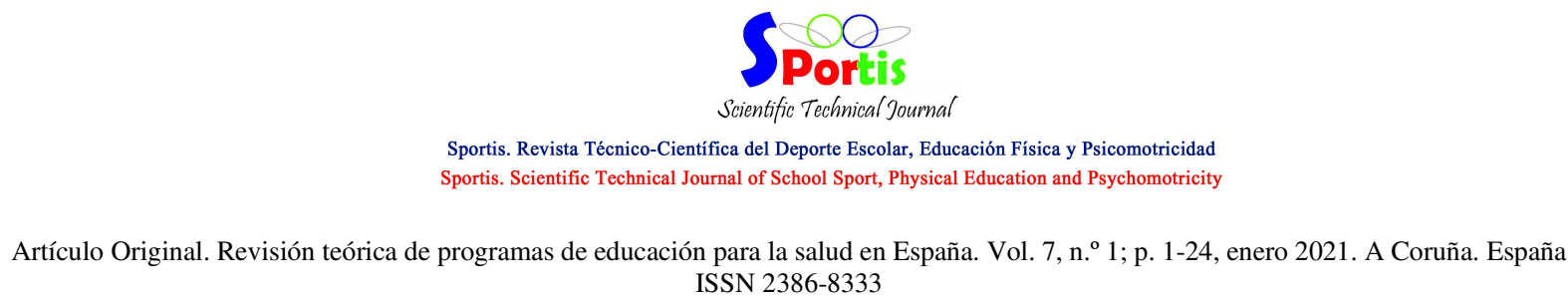
ISSN 2386-8333

Es en la etapa escolar cuando se aprenden los hábitos alimentarios que se desarrollarán posteriormente durante el resto de la vida de la persona (Macedo, Valverde, López, Rodríguez, López, Delgado, et al. 2007; Tojo y Leis, 2007) y donde se cimientan las bases para los pasos posteriores, donde se tiene en cuenta una nutrición correcta que favorezca el crecimiento y el desarrollo (Ortiz-Andrellucchi, Peña, Albino, Mönckeberg y Serra, 2006).

Según estudios realizados por Fraile y De Diego (2006) la práctica deportiva en la escuela es considerada tanto por niños como por entrenadores beneficiosa para la salud y por lo tanto es el motivo más substancial por el que lo hacen. Además, está inmersa en una expresión cultural, educativa y preventiva donde se conciben hábitos saludables para el futuro.

Pero a principios de la adolescencia la frecuencia con la que se realiza actividad física disminuye llegando a cifras que preocupan, ya que a los 14 años la mitad de las adolescentes no suelen realizar actividad física o deportiva fuera del horario lectivo (González, Sarría y Coca, 2004; Martín, Tercedor, Pérez, Chillón y Delgado, 2004).

No todos los escolares que comienzan a hacer deporte lo siguen haciendo para siempre o al menos lo tienen presente en sus vidas continuamente. Tanto los chicos como las chicas, sin distinción, cambian de deporte o dejan de hacerlo porque ya no les aporta lo que antes, o no disponen ya del tiempo de ocio suficiente o ven incrementada la dificultad de seguir accediendo a la práctica de ese mismo deporte (Camacho, 2003; Gómez, 2001; Vázquez, Fernández, Ferro, Learreta y Viejo, 2000).

Por otra parte, en referencia a los hábitos alimentarios los padres deben decidir la cantidad y calidad de los alimentos proporcionados a sus hijos debido a la gran influencia que tienen sobre los hábitos alimentarios de los niños. Padres y escuela (sobre todo personal docente) son vitales en promover hábitos de alimentación saludables y que los niños los adquieran para toda su vida, con la ayuda de la promoción y la Educación para la salud (Estevan, 2004).

Y aunque es el círculo familiar, la primera toma de contacto con los hábitos alimenticios por la elevada influencia en la dieta de los pequeños y en sus directrices correspondientes a la alimentación (Moreno y Galiano, 2006) es en la etapa escolar donde el propio centro educativo está en posesión de un papel primordial en el impulso de factores que

Para citar este artículo utilice la siguiente referencia: Salvador, F.; Muros, J.J.; Gámiz, V.Ma.; Zurita, F. (2021). Revisión teórica de programas de educación para la salud en España. Sportis Sci J, 7 (1), 1-24. https://doi.org/10.17979/sportis.2021.7.1.6530 http://revistas.udc.es/ 


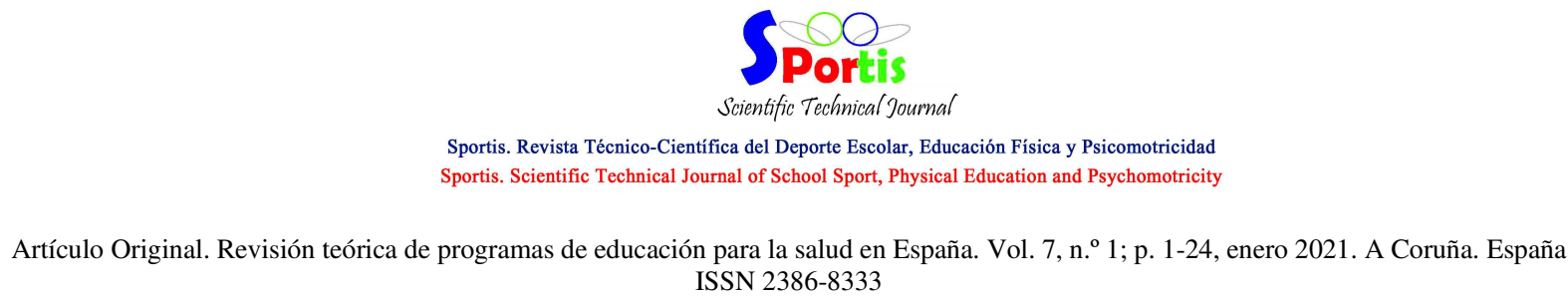

salvaguarden los hábitos alimentarios, siendo esencial el diseño de programas que ayuden a impulsar hábitos alimentarios saludables y por lo tanto, como consecuencia más inmediata, a crear estilos de vida saludables (Macías, Gordillo y Camacho, 2012).

Según estudios realizados como el de Chacón, Muros, Cachón, Zagalaz, Castro y Zurita (2018) existe una relación positiva entre el grado de adherencia a la dieta mediterránea y el grado de actividad física en escolares tanto en la etapa primaria como en la adolescencia. Y si existen en gran medida tanto dicha adherencia como un alto nivel de actividad física repercutirá directa y positivamente en la calidad de vida relacionada con la salud (Evaristo et. al, 2018).

Por tanto, en edad infantil que es cuando la persona está en pleno desarrollo y tiene que seguir haciéndolo, todo apunta a que seguir una dieta saludable y adecuada podrá optimizar el potencial genético que hay en el niño. Y puede cobrar una mayor importancia dar prioridad a la alimentación saludable sobre la alimentación enfocada solamente al aporte de energía para realizar actividad física.

Durante la etapa escolar es relevante establecer hábitos de alimentación saludables porque es en la infancia temprana donde se adquieren (Brug, Tak, te Velde, Bere y de Bourdeaudhuij, 2008) que pueden continuar en la etapa adulta de una persona (Craigie, Lake, Kelly, Adamson y Mathers, 2011).

Arriscado et al. (2014) estudiaron la adherencia a la DM en relación al tiempo que se pasa frente a la pantalla y las horas de sueño por la noche en días lectivos. Se concluyó que los escolares que tenían hábitos de alimentación poco saludables presentaban al mismo tiempo sedentarismo. El hecho de estar frente a una pantalla les incita en mayor medida a ingerir alimentos con elevado contenido en grasas y azúcares, ya que resulta más cómodo hacerlo frente a esa pantalla y además cabe la posibilidad de estar influido por la publicidad aparecida.

Al mismo tiempo, se encontraron vínculos entre los patrones de dieta mediterránea y las horas nocturnas de descanso. Sin embargo, en los chicos la cantidad de sueño nocturno se ve afectada negativamente por el tiempo que dedican a estar delante de la pantalla.

Un estudio publicado por Vareiro et al. (2009) tuvo como objetivo ver cómo cuarenta y un países habían mostrado en los últimos cuarenta años adherencia a los patrones marcados

Para citar este artículo utilice la siguiente referencia: Salvador, F.; Muros, J.J.; Gámiz, V.Ma .; Zurita, F. (2021). Revisión teórica de programas de educación para la salud en España. Sportis Sci J, 7 (1), 1-24. https://doi.org/10.17979/sportis.2021.7.1.6530 http://revistas.udc.es/ 


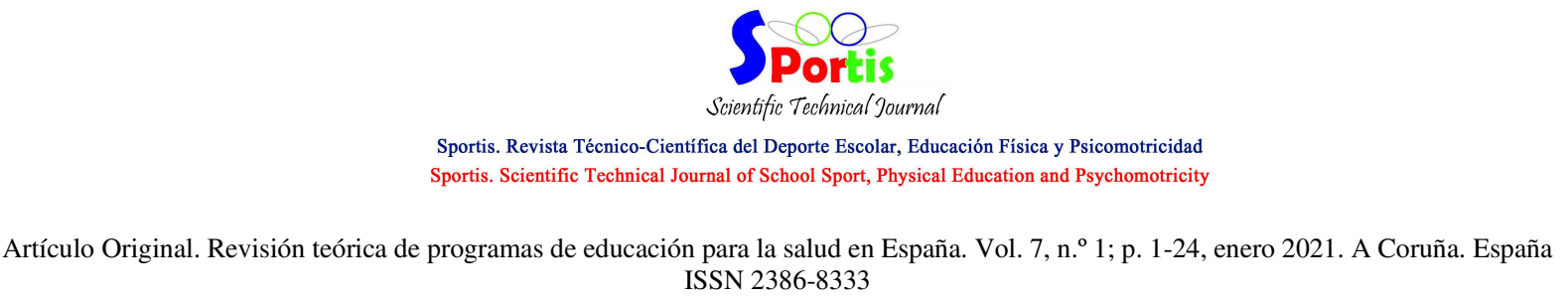

por la DM. Son los países del Mediterráneo pertenecientes a Europa los que más disminuyen en cuanto a la adherencia. España ocupa el cuarto lugar en cuanto al que más se aleja de esa DM. En contraposición, los países del Norte de Europa tienen la tendencia contraria ya que presentan un aumento en cuanto a la presencia de alimentos mediterráneos y a su vez, dejan de consumir los propiamente tradicionales de su zona.

Si se establece una relación directa entre el sedentarismo y la dieta basándonos en Pearson y Biddle (2011) podemos afirmar que el tiempo que pasan niños y adolescentes delante de la televisión influye para aumentar el consumo de comida rápida, aperitivos de alta intensidad, bebidas de alta densidad de energía, con un mayor consumo de energía total y al mismo tiempo, existe una mayor energía obtenida de las grasas. Además, influye negativamente en el consumo de verdura y fruta. Todo esto se acentúa aún más en el género masculino que en el femenino.

Un reciente estudio piloto de El CoCo (2019) que analiza la calidad nutricional, el grado de procesamiento y los aditivos de los productos de las marcas más vendidas ha servido para comprobar que más del $50 \%$ de los alimentos que consumen en casa los españoles presentan un elevado grado de procesamiento además de ser abundantes en grasas saturadas, sal y azúcares libres. Por otro lado, no son precisamente ricos en fibra, proteínas, vitaminas o minerales. En cuanto a los niveles de azúcares añadidos también más del 50\% tenía en su lista de ingredientes uno o más tipos de azúcares. El consumir con asiduidad elevadas cantidades de azúcares añadidos está directamente relacionado con obesidad, problemas cardiovasculares o diabetes (entre otros), lo que representa un problema añadido al sedentarismo.

Por tanto, se plantea el siguiente trabajo con los objetivos de: a) realizar una revisión teórica de artículos relacionados con programas de salud y b) localizar y analizar un programa de salud en cada una de las provincias de España.

\section{Metodología}

Se realizó una revisión de la literatura en las bases de datos: Web of Science, SCOPUS y Pubmed, para ello se tuvieron en cuenta tres criterios de inclusión:

- Estudios realizados en España.

- Que fuesen de los 10 últimos años.

- Que se desarrollase un programa de intervención.

Para citar este artículo utilice la siguiente referencia: Salvador, F.; Muros, J.J.; Gámiz, V.Mª.; Zurita, F. (2021). Revisión teórica de programas de educación para la salud en España. Sportis Sci J, 7 (1), 1-24. https://doi.org/10.17979/sportis.2021.7.1.6530 http://revistas.udc.es/ 
Para la búsqueda se incluyeron los descriptores de: "Programme Intervention",

"Health", "Adolescent" y "Child"

En cada una de las provincias se intentó localizar un par de artículos, y de ellos se seleccionó el que los investigadores consideraron más oportuno para este estudio de investigación.

Se establece representación del diagrama seguido, donde se explican las cuatro fases desarrolladas en este estudio:

Figura 1. Diagrama de estructuración de las fases de la revisión de la literatura

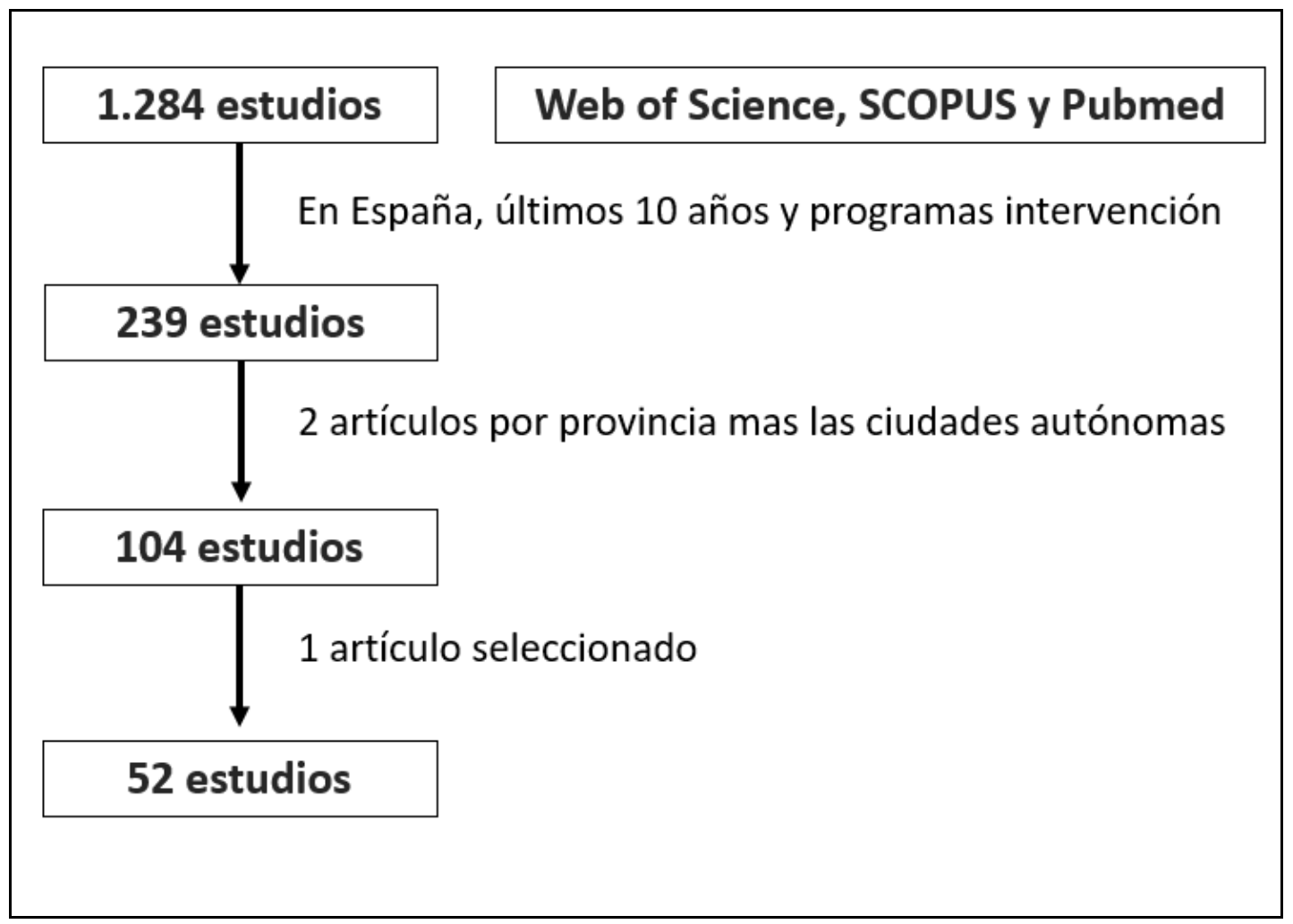

\section{Resultados y Discusión}

En lo que se refiere a los programas de educación para la salud y su abordaje en España se tienen los siguientes casos distribuidos en cada una de las comunidades autónomas de España, de esta manera en la Comunidad Autónoma de Andalucía, González-Jiménez, Cañadas, Lastra-Caro y Cañadas-De la Fuente (2015) en Almería, indican que tras la intervención se produce un descenso en el índice de masa corporal. En el caso de su estudio por sexos, se puede observar que en las chicas hay valores superiores.

Para citar este artículo utilice la siguiente referencia: Salvador, F.; Muros, J.J.; Gámiz, V.Ma.; Zurita, F. (2021). Revisión teórica de programas de educación para la salud en España. Sportis Sci J, 7 (1), 1-24. https://doi.org/10.17979/sportis.2021.7.1.6530 


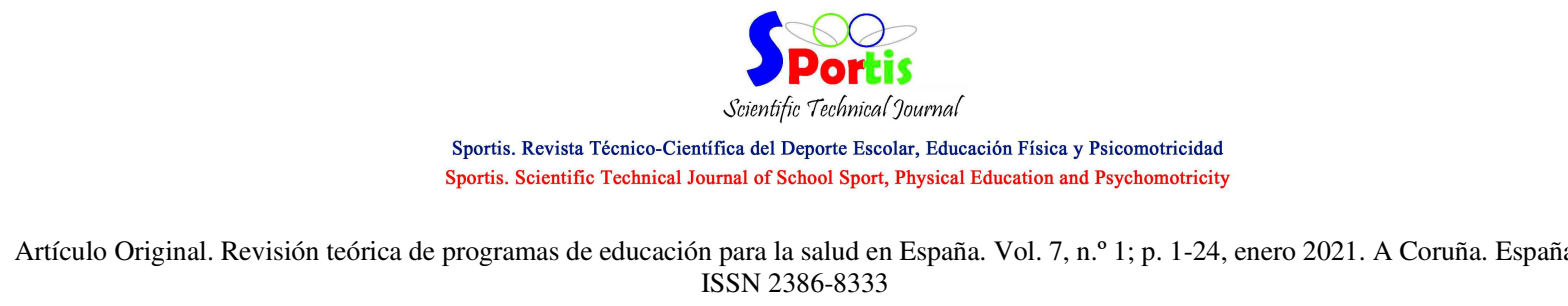

Los alumnos pasaron de un nivel nutricional muy bajo antes de la intervención, hasta un nivel nutricional medio después, en Cádiz, Molinero, Castro-Piñero, Ruiz, GonzálezMontesinos, Mora y Márquez (2010), existían diferencias entre ambos sexos en cuanto al porcentaje de aquellos que afirmaban no practicar nunca deporte, mayor en el caso de las chicas. Una parte importante de los alumnos no desayunaba todos los días de la semana.

Cerca de una cuarta parte reconocía no consumir nunca verduras u hortalizas, más al norte Martín-Martínez, Chirosa-Ríos, Reigal, Hernández-Mendo, Juárez-Ruiz de Mier y Guisado-Barrilao (2015), en Córdoba, los resultados obtenidos en esta investigación indican que el programa de actividad física aplicado ha tenido un efecto positivo sobre el Índice de Masa Corporal y la condición física de los participantes.

En Granada, Muros, Zabala, Oliveras-López, Ocaña-Lara y López-García de la Serra (2013), los resultados de este estudio demuestran que un programa de 7 semanas que incorpora vigorosa actividad física de corta duración y un componente de educación nutricional realizado en la escuela puede mejorar los parámetros relacionados con la salud en los niños, en Huelva, Robles, Obando, González y Bueno (2011), en cuanto al hábito de ejercicio físico, afirmaron haber realizado algún tipo de actividad física fuera del horario escolar la semana anterior a la encuesta más del $75 \%$ de los varones y la mitad de las mujeres.

En Jaén, Torres-Luque, Carpio, Lara y Zagalaz (2014), los resultados muestran unas características de composición corporal consideradas como normales para la población infantil, existiendo escasas diferencias en cuanto al nivel de actividad física. Sí se observan diferencias en cuanto a la capacidad cardiorrespiratoria. Se observan diferencias respecto al género en cuanto a la potencia de piernas, siendo mayor en los chicos que en las chicas.

A su vez, las chicas obtienen mayores valores de flexibilidad, en la ciudad de Málaga, Sánchez-Rivas, Mayorga-Vega, Fernández-Rodríguez y Merino-Marbán (2014), señalan en su programa de estiramiento estático pasivo de la musculatura isquiosural de tres minutos de duración en cada sesión de EF, parece tener un efecto positivo sobre los resultados del IMC en escolares de Educación Primaria, y por último en Sevilla, Oliva, Tous, Gil, Longo, Pereira y García (2013), después de la intervención educativa los alumnos del grupo de intervención poseen más conocimientos relativos a alimentación y hábitos de vida saludables que los alumnos del grupo control, y esta diferencia es estadísticamente significativa.

Para citar este artículo utilice la siguiente referencia: Salvador, F.; Muros, J.J.; Gámiz, V.Ma.; Zurita, F. (2021). Revisión teórica de programas de educación para la salud en España. Sportis Sci J, 7 (1), 1-24. https://doi.org/10.17979/sportis.2021.7.1.6530 http://revistas.udc.es/ 


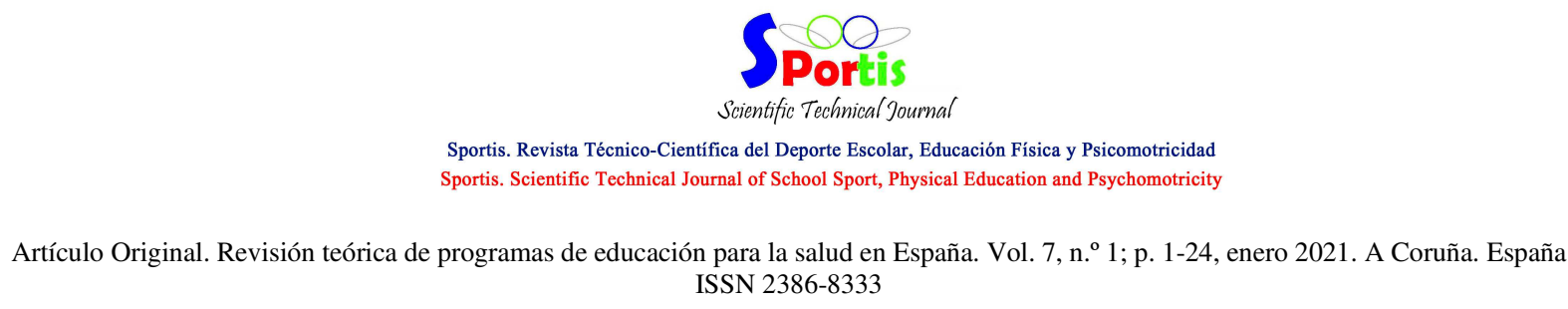

En Aragón, en la ciudad de Huesca, Álvarez-Medina et al. (2013), desarrollan un programa el cual ha sido efectivo para cortar la tendencia ascendente del peso. Se ha producido un cambio hacia un estilo de vida saludable, abordado desde una intervención educativa interdisciplinar, atendiendo a aspectos nutricionales, biomédicos y de actividad física. Los hombres comen peor que las mujeres.

En Teruel y Zaragoza, Casajús, Ortega, Vicente-Rodríguez, Leiva, Moreno y Ara (2012), se obtienen valores normales de condición física. Un 9,1\% de los chicos y 4,8\% de las chicas presenta riesgo fututo de salud sobre la base de su condición aeróbica. Mejor condición aeróbica se asocia con cantidades significativamente menores de grasa subcutánea total y en el tronco.

En Asturias, en la ciudad de Oviedo, Pérez-Solís, Díaz-Martín, Álvarez-Caro, SuárezTomás, Suárez-Menéndez y Riaño-Galán (2015), se indica en un programa titulado: “Tú decides tu salud. ¡Ponte a vivir!”, que este programa escolar consiguió pequeñas mejoras en el IMC y la calidad de la dieta.

En las Islas Baleares, Puig, Moñino, Colomer, Martí y Muñoz-Octavio de Toledo (2006), en la ciudad de Mallorca, el patrón femenino mejora más que el masculino ( $\mathrm{p}<0,001$ ), especialmente en el grupo de 12 - 15 años. Aumenta el porcentaje de Dieta Mediterránea Óptima $(\mathrm{p}<0,001)$ y el $29,6 \%$ de los escolares refieren haber modificado sus hábitos a partir de las intervenciones.

En las Islas Canarias, los investigadores Ruiz-Santana, Ruiz-Hernández, HernándezSocorro, Álvarez-Falcón y Saavedra-Santana (2009), en Las Palmas, indican con su programa educacional que el porcentaje de niños con peso ideal según el IMC fue del 51,9\% y ocho $(15,4 \%)$ son obesos, y en Santa Cruz de Tenerife, Calvo-Pacheco, Moreno, RodríguezÁlvarez, Abreu, Álvarez-Marante y Arias (2015), señalan con sus programas de charlas que la mayoría de los escolares, con ligero incremento después de la intervención educativa, conocen la importancia del desayuno, sin existir diferencias según sexo.

En Cantabria, en la ciudad de Santander, Tercedor et al. (2007), los niveles de práctica de actividad física son bajos, siendo menores aún en el caso de las chicas. El consumo de tabaco muestra relación inversa con la práctica de actividad física, resultando los sujetos más activos físicamente los menos consumidores de tabaco.

Para citar este artículo utilice la siguiente referencia: Salvador, F.; Muros, J.J.; Gámiz, V.Ma.; Zurita, F. (2021). Revisión teórica de programas de educación para la salud en España. Sportis Sci J, 7 (1), 1-24. https://doi.org/10.17979/sportis.2021.7.1.6530 http://revistas.udc.es/ 


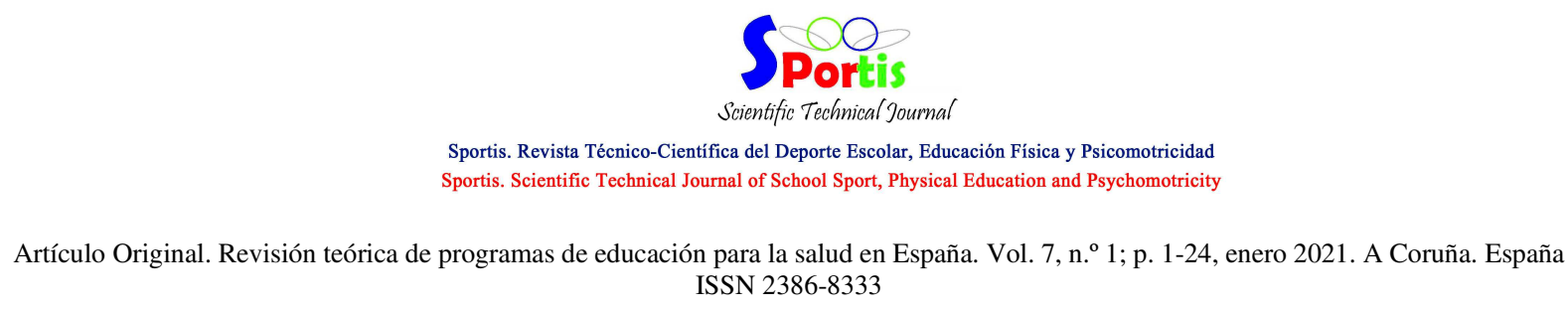

En Castilla La-Mancha, en Albacete, los investigadores Contreras et al. (2010), los adolescentes que practicaban habitualmente algún deporte tenían percepciones superiores de su autoconcepto, tanto general como físico. Los practicantes presentaron puntuaciones más altas en todas las escalas del CAF. Además, también se encontró que la frecuencia con la que se practicaba el deporte, la duración de la práctica, los años de dedicación, la satisfacción y el gusto por la práctica deportiva se relacionaba positivamente con el autoconcepto físico y general, en Ciudad Real, Calatayud-Sáez, Calatayud-Moscoso del Prado y Gallego-Fernández (2011), establecen en su programa de "Aprende a comer" que se manifiesta la disminución del percentil del índice de masa corporal, con un descenso importante de niños con sobrepeso y obesidad, así mismo el porcentaje de masa grasa corporal disminuyó de forma significativa, y el índice de calidad nutricional KidMed fue óptimo en el 95\% al finalizar la intervención.

Unos años antes Martínez-Vizcaíno et al. (2008), en Cuenca señalan en su programa de actividad física recreativa se redujo la adiposidad y aspectos antopométricos de la muestra de estudio. En este sentido Fernández-Morales, Aguilar-Vilas, Mateos-Vega y Martínez-Para (2008), en Guadalajara señalan que la población estudiada consume un desayuno poco adecuado lo que puede afectar a sus resultados académicos, especialmente de algunas asignaturas, y por último en Toledo los investigadores Martínez-Martínez, Contreras, Aznar y Lera (2012), señalan que no hay diferencias estadísticas en los escolares con o sin problemas de coordinación en base al autoconcepto.

En Castilla y León, en un estudio en las ciudades de Ávila, Salamanca y Zamora, Morán y Rivera (2015), hacen un recorrido bibliográfico sobre lo señalado en la legislación respecto a los comedores escolares, del mismo modo en Burgos, Gutiérrez-Abad, San José, Castroviejo, Sánchez y Cordero (2015), señalan en su estudio transversal en el que se han obtenido datos de una muestra aleatoria de niños escolarizados entre segundo y quinto curso de Educación Primaria Obligatoria en la ciudad de Burgos mediante la cumplimentación de una encuesta, que la prevalencia de enuresis es del 7,3\%., siendo más frecuente en varones, y que se asocian a trastornos del sueño y del comportamiento y con bajo rendimiento escolar.

En León, Calleja-Fernández et al. (2011), en un programa sobre alimentación señalan que la modificación de los hábitos dietéticos del almuerzo de los escolares es posible con campañas sencillas de intervención nutricional. En la ciudad de Palencia, los investigadores

Para citar este artículo utilice la siguiente referencia: Salvador, F.; Muros, J.J.; Gámiz, V.Mª.; Zurita, F. (2021). Revisión teórica de programas de educación para la salud en España. Sportis Sci J, 7 (1), 1-24. https://doi.org/10.17979/sportis.2021.7.1.6530 http://revistas.udc.es/ 


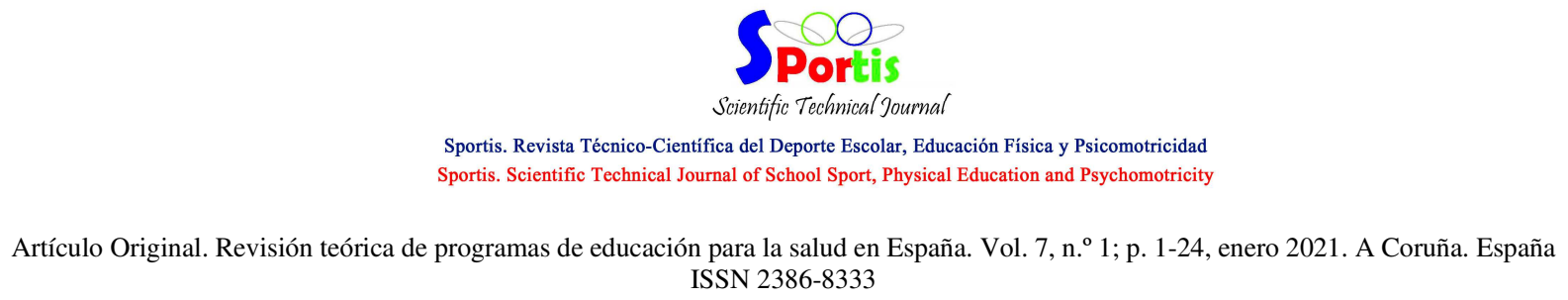

Cano-Garcinuño, García, Alonso y López (2011), señalan que la actividad física en la población estudiada es aceptable, pero hay diferencias entre sexos y tiende a disminuir durante la adolescencia.

En Segovia, González-Pascual, Manrique-Arribas y López-Pastor (2012), señalan que en la aplicación del programa PIDEMSG, en lo relativo a las actitudes y valores respecto a la actividad física y deporte, todos los agentes implicados muestran un discurso claramente orientado hacia la participación y la educación. Más al este en la ciudad de Soria, PérezGallardo, Bayona, Mingo y Rubiales (2011), indican que la proporción de escolares que presentan una adhesión media-alta a los patrones de la dieta mediterránea es superior en los encuestados de años posteriores.

En Valladolid, Ramos (2013), expone una investigación sobre el desplazamiento activo, entendiendo este como el traslado andando o en bicicleta a lugares cotidianos, enfocado a ponerlo en práctica en el camino a la escuela. Se analizan las ventajas e inconvenientes del desplazamiento activo, así como se profundiza en el proyecto llamado "Caminos escolares seguros".

En Cataluña, los investigadores Babiloni et al. (2017), en la ciudad de Barcelona se plantea que el programa de educación nutricional planteado tiene una incidencia positiva sobre la calidad de la dieta y la prevalencia de sobrepeso y obesidad en la población infantil, en Girona, los autores Oviedo et al. (2013), señalan que los resultados de este estudio nos indican que los adolescentes dedican poco tiempo a la realización de actividad física. Los principales resultados muestran que los varones realizan actividad física durante más tiempo y a mayores intensidades que las mujeres.

En Lleida, Serra-Paya, Ensenyat-Solé y Blanco-Nespereira (2014), señalan que existe un cambio positivo en los hábitos de actividad física, las conductas sedentarias y la puntuación del IMC en niños poco activos con sobrepeso y obesidad. Esta intervención podría ser una interesante herramienta en salud pública para el tratamiento de la obesidad infantil, y finalmente en Tarragona, los investigadores Llauradó, Tarro, Moriña, Queral, Giralt y Solà (2014), señalan que tras los 22 meses, la prevalencia de obesidad y los valores de IMC fueron similares en los grupos de intervención y control. En relación con los niños de las escuelas de control, el porcentaje de niños en el grupo de intervención que realizaron $\geq 4$ PA / semana de

Para citar este artículo utilice la siguiente referencia: Salvador, F.; Muros, J.J.; Gámiz, V.Mª.; Zurita, F. (2021). Revisión teórica de programas de educación para la salud en España. Sportis Sci J, 7 (1), 1-24. https://doi.org/10.17979/sportis.2021.7.1.6530

http://revistas.udc.es/ 
educación después de la escuela fue $15 \%$ mayor, mientras que el porcentaje de niñas en ambos grupos permaneció similar.

En la Ciudad Autónoma de Ceuta, Nieto-García, Nieto-García, Lacalle-Remigio y Abdel-Kader (2001), indican que los índices de salud bucodental se encuentran en decadencia, mostrándose valores altos en problemas con las caries, y en la Ciudad Autónoma de Melilla, Granda-Vera, Montilla, Barbero, Mingorance y Alemán (2010), los resultados muestran que los alumnos declaran realizar actividad físico-deportiva en su tiempo libre ("Más de 3 veces por semana ") en un 38,3\%, mientras que los chicos manifiestan dedicar más tiempo diario a la práctica de actividad físico-deportiva (2 horas o más).

En Extremadura, en la ciudad de Badajoz, los autores Sancho-González, PérezPatrón, Torres-Asensio y Campillo-Álvarez (2002), en el estudio planteado reflejan que los jóvenes ingieren una dieta alejada de las recomendaciones internacionales y desarrollan hábitos nocivos para la salud como: tabaquismo, consumo de alcohol y elevados índices de sedentarismo; más al norte en Cáceres, García-Hermoso, Escalante, Domínguez, Saavedra (2013), en la implementación de su programa de actividad física revelan que tras la intervención se observaron cambios en el IMC, dejando de ser obesos tras la intervención y mejorando los niveles de actividad física, mostrándose que intervenciones longitudinales generan beneficios positivos en niños obesos.

En el País Vasco, los investigadores Lafuente, Gómez-Pérez de Mendiola, Aguirre, Zabala-Galán, Irurzun-Zuazabal y Gorritxo-Gil (2002) en Álava, evalúan los determinantes de salud oral en adolescentes. El consumo de pastelería industrial y de golosinas es habitual. Los hábitos de salud oral presentan deficiencias, en Bilbao los autores Zamacona, RocandioPablo, Municio, Ansotegui-Alday, Martínez de la Pera y Arroyo-Izaga (2011), los niños/as estudiados presentan un elevado consumo de alimentos ricos en azúcar o sal, así como diferencias significativas en el consumo de dichos productos según grupos de edad, y en San Sebastián los autores Iturbe-Lete y Perales-Antón (2002), tras la implementación de su programa se indica que el 36\% de los jóvenes opinan que la calidad de su dieta ha mejorado, y el $85 \%$ conoce el modelo dietético equilibrado.

En Galicia en la ciudad de A Coruña, Morenilla y Iglesias (2012), señalan que el IMC de los sujetos indica un comportamiento similar por edades y percentiles, a los valores de 


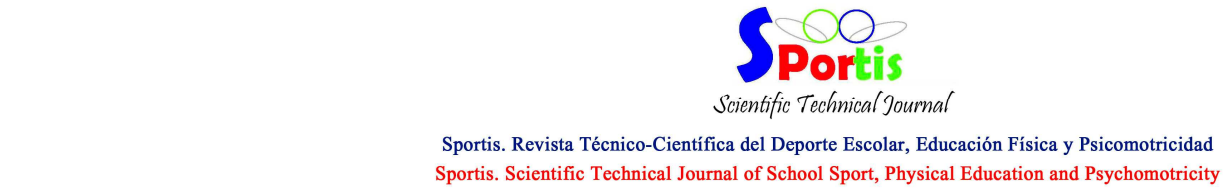

Artículo Original. Revisión teórica de programas de educación para la salud en España. Vol. 7, n. ${ }^{\circ}$ 1; p. 1-24, enero 2021. A Coruña. España ISSN 2386-8333

referencia de la población adolescente gallega y española. La muestra del estudio indicó niveles similares en resistencia cardiorrespiratoria en varones y en mujeres. Los niveles de flexibilidad en las chicas de la muestra fueron inferiores que los planteados para las mujeres adolescentes españolas, en Ourense los autores García, Míguez y De la Montaña (2011), se indicó que el 38,7\% presentó sobrepeso u obesidad; aunque la prevalencia fue mayor en niñas. El 76,6\% fueron inactivos o muy inactivos, con un gasto energético medio de 33,41 METS

En Pontevedra, Lois-Carro y Rial-Rebullido (2016), destacan en sus resultados que un $32 \%$ no realiza desayuno a media mañana y un 10,6\% del alumnado posee sobrepeso. El nivel de actividad física es más alto en niños que niñas, y en Lugo, Muñoz Heras, FernándezPedraza y Navarro Patón (2015), indican como resultados destacables, se ha obtenido que un bajo porcentaje realizan las 5 ingestas diarias recomendadas. No realizan actividad física durante el recreo escolar ni en el tiempo libre antes o después del comedor escolar. El 16,67\% de los niños y niñas sufre sobrepeso.

En la Comunidad Autónoma de Madrid, los autores Laguna, Lara y Aznar (2011), revelaron que las niñas, realizaron significativamente menos actividad física que los niños. Todos los niños y niñas realizaron significativamente más actividad física durante los días de fin de semana que durante los días laborables.

En la Región de Murcia, López-Sánchez, López y Díaz-Suárez (2016), en su estudio tras la intervención se han encontrado mejoras significativas en la masa grasa de los escolares y el IMC. Estas mejoras han sido significativas en los chicos y en las chicas.

En Navarra, concretamente en Pamplona, Castillo-Alvira, Rodríguez-Negro y YanciYrigoyen (2016), en su trabajo plantean que la unidad didáctica de fuerza, que consistía en 16 sesiones con una duración de 60 minutos por sesión y que se realizaron en 8 semanas consecutivas, generó una mejora en la capacidad de LBM en los chicos y las chicas de primero de bachillerato.

En La Rioja, en la ciudad de Logroño, Arriscado et al. (2015), los alumnos de género masculino, quienes practicaban actividades deportivas extraescolares y los que se encontraban en un estadio de desarrollo menor reportaron mayores niveles de actividad física. Se

Para citar este artículo utilice la siguiente referencia: Salvador, F.; Muros, J.J.; Gámiz, V.Ma.; Zurita, F. (2021). Revisión teórica de programas de educación para la salud en España. Sportis Sci J, 7 (1), 1-24. https://doi.org/10.17979/sportis.2021.7.1.6530 http://revistas.udc.es/ 


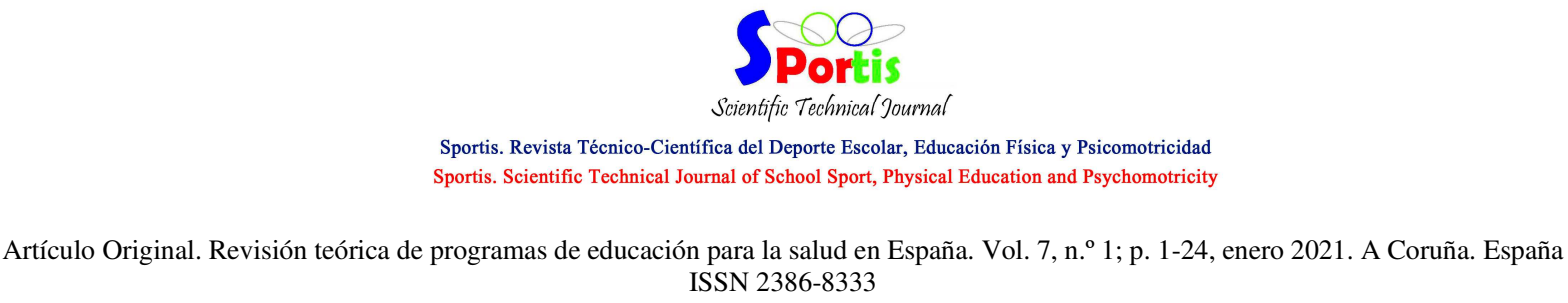

encontraron correlaciones directas entre la práctica y la condición física, especialmente con la capacidad aeróbica.

En la Comunidad Autónoma de Valencia, los autores Martínez-García y TrescastroLópez (2016), en Alicante indican en su trabajo de almuerzo saludable que la intervención educativa sobre alimentación y nutrición produce cambios positivos sobre la población, mejorando el conocimiento y consumo de alimentos saludables. Por ello, se puede afirmar que los programas de educación alimentaria y nutricional son necesarios en la sociedad actual para prevenir el exceso de peso y promover hábitos saludables.

Más al norte en Castellón, los científicos Pin-Arboledas, Alarcón, González, Roselló y Salort (2011), indican que los malos hábitos de sueño son prevalentes. Es necesario incrementar las medidas de educación transmitiendo la importancia de las horas de sueño y finalmente en Valencia, Martínez, Hernández, Ojeda, Mena, Alegre y Alfonso (2009), revelan tras su programa que el 37,8\% de los adolescentes ha mejorado el grado de la calidad de la dieta. Aquellos que consumen una dieta de muy buena calidad pasando del 30,0\% al 58,6\%. También es significativo el número de alumnos que han comenzado a desayunar y los que han dejado de tomar bollería industrial en esta ingesta.

\section{Conclusiones y limitaciones}

Como principal conclusión señalar que los programas de salud se hallan reflejados en todas las provincias de España y en todos los casos se ha detectado al menos un estudio en los últimos diez años

Es interesante destacar como en todas las provincias de España hay un interés por determinar aspectos relacionados con la salud.

Como principal limitación se debe destacar que se abordaron diversos temas de investigación, y no se centró exclusivamente en uno de ellos.

\section{Referencias bibliográficas}

Álvarez-Medina, J., Rodríguez, J. S. F., Marqueta, P. M., Salillas, L. G., Correas, R. G., Costa, S. L., y Julián, C. A. (2013). Desarrollo de un programa de modificación de

Para citar este artículo utilice la siguiente referencia: Salvador, F.; Muros, J.J.; Gámiz, V.Må.; Zurita, F. (2021). Revisión teórica de programas de educación para la salud en España. Sportis Sci J, 7 (1), 1-24. https://doi.org/10.17979/sportis.2021.7.1.6530 http://revistas.udc.es/ 


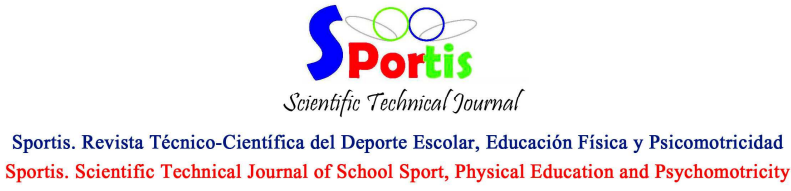

Artículo Original. Revisión teórica de programas de educación para la salud en España. Vol. 7, n. ${ }^{\circ}$ 1; p. 1-24, enero 2021. A Coruña. España ISSN 2386-8333

conductas en población infantil obesa deportista. Apunts. Educació física i esports, 111, 15-22. https://doi.org/10.5672/apunts.2014-0983.es.(2013/1).111.01

Aranceta, J. (2001). Spanish food patterns. Public Health Nutrition, 4(6a), 1399-1402.

Arriscado, D., Muros, J. J., Zabala, M., y Dalmau, J. M. (2014). Relación entre condición física y composición corporal en escolares de primaria del norte de España (Logroño). Nutrición Hospitalaria, 30(2), 385-394.

Babiloni, M., et al. (2017). Mejora de la calidad de la dieta y del estado nutricional en población infantil mediante un programa innovador de educación nutricional: INFADIMED. $\quad$ Gaceta $\quad$ Sanitaria, $12 \quad$ (IN-PRESS). https://doi.org/10.1016/j.gaceta.2016.10.013

Bacardí-Gascón, M., Jiménez-Cruz, A., Jones, E., y Guzmán González, V. (2007). Alta prevalencia de obesidad y obesidad abdominal en niños escolares entre 6 y 12 años de edad. Bol Med Hosp Infant Mex, 64(6), 362-369 https://doi.org/10.20960/nh.565

Brug, J., Tak, N. I., te Velde, S. J., Bere, E., y De Bourdeaudhuij, I. (2008). Taste preferences, liking and other factors related to fruit and vegetable intakes among schoolchildren: Results from observational studies. British Journal of Nutrition, 99(S1), S7-S14. https://doi.org/10.1017/s0007114508892458

Calatayud-Sáez, F., Calatayud-Moscoso del Prado, B., y Gallego-Fernández-Pacheco, J. G. (2011). Efectos de una dieta mediterránea tradicional en niños con sobrepeso y obesidad tras un año de intervención. Pediatría Atención Primaria, 13(52), 553-569. https://doi.org/10.4321/s1139-76322011000600005

Calleja Fernández, A., Muñoz Weigand, C., Ballesteros Pomar, M., Vidal Casariego, A., López Gómez, J. J., Cano Rodríguez, I., ... y García Fernández, M. (2011). Modificación de los hábitos alimentarios del almuerzo en una población escolar. Nutrición Hospitalaria, 26(3), 560-565. https://doi.org/10.20960/nh.22

Calvo-Pacheco, M., Moreno, P., Rodríguez-Álvarez, C., Abreu, R., Álvarez-Marante, R., y Arias, Á. (2015). Evaluación de una intervención educativa sobre los conocimientos de los escolares de la alimentación saludable. Higiene y Sanidad Ambiental, 15(2), 1295-1301.

Camacho, A. S. (2003). La investigación sobre el pensamiento del alumnado. Una revisión desde la educación física. Revista de Educación, 331, 577-613. https://doi.org/10.35537/10915/65579

Cano-Garcinuño, A., García, I. P., Alonso, I. C., y López, S. A. (2011). Determinantes del nivel de actividad física en escolares y adolescentes: estudio OPACA. Anales de Pediatría, 74(1), 15-24. https://doi.org/10.1016/j.anpedi.2010.08.009

Carvajal, C., y Rodríguez, M. (1999). La escuela, la familia y la educación para la salud. La Habana.

Casajús, J.A., Ortega, F.B., Vicente-Rodríguez, G., Leiva, M.T., Moreno, L.A. y Ara, I. (2012). Condición física, distribución grasa y salud en escolares aragoneses (7 a 12 Para citar este artículo utilice la siguiente referencia: Salvador, F.; Muros, J.J.; Gámiz, V.Ma .; Zurita, F. (2021). Revisión teórica de programas de educación para la salud en España. Sportis Sci J, 7 (1), 1-24. https://doi.org/10.17979/sportis.2021.7.1.6530 http://revistas.udc.es/ 

ISSN 2386-8333

años). Revista Internacional de Medicina y Ciencias de la Actividad Física y el Deporte, 12(47), 523-537. https://doi.org/10.15366/rimcafd2018.72.004

Casas, S. B., y Klijn, T. P. (2006). Promoción de la salud y su entorno laboral saludable. Revista Latinoamericana Enfermagem, 14(1), 136-141. https://doi.org/10.1590/s010411692006000100019

Castillo-Alvira, D., Rodríguez Negro, J., y Yanci Yrigoyen, J. (2016). Influencia de una unidad didáctica de fuerza en el rendimiento de lanzamiento de balón medicinal en alumnos de bachillerato. Sportis, 2(3),

343-355. https://doi.org/10.17979/sportis.2016.2.3.1727

Chacón, R., Muros, J.J., Cachón, J., Zagalaz, M.L., Castro, M., y Zurita, F. (2018). Actividad física, dieta mediterránea, capacidad aeróbica y clima motivacional hacia el deporte en escolares de la provincia de Granada: un modelo de ecuaciones estructurales. Nutr Hosp, 35, 774-81. https://doi.org/10.20960/nh.1511

Contreras, O.R., Fernández, J.G., García, L.M., Palou, P., y Ponseti, J. (2010). El autoconcepto físico y su relación con la práctica deportiva en estudiantes y adolescentes. Revista de Psicología del Deporte, 19 (1), 23-39. https://doi.org/10.36443/10259/4489

Craigie, A. M., Lake, A. A., Kelly, S. A., Adamson, A. J., y Mathers, J. C. (2011). Tracking of obesity-related behaviours from childhood to adulthood: A systematic review. Maturitas, 70(3), 266-284. https://doi.org/10.1016/j.maturitas.2011.08.005

Devís Devís, J., y Garde Cantera, M. A. (2002). La promoción de la actividad física relacionada con la salud en el ámbito escolar: Implicaciones y propuestas a partir de un estudio realizado entre adolescentes. Apunts: Educación Física y Deportes, (67), 54-63. https://doi.org/10.5672/apunts.2014-0983.es.(2019/3).137.03

Dietz, W. H. (1998). Health consequences of obesity in youth: Childhood predictors of adult disease. Pediatrics, 101(2), 518-525.

Durá, T. (2001). Ingesta de energía y nutrientes en los alumnos de educación secundaria obligatoria. Anales de Pediatría, 54(6) 547-554. https://doi.org/10.1016/s16954033(01)77592-3

El CoCo, E. (2019) Una aproximación a la cesta de la compra de los españoles: Estudio piloto que analiza la calidad nutricional, el grado de procesamiento y los aditivos de los productos de las marcas más vendidas. https://doi.org/10.15765/wpmgem.v1i2.857.s84

Erickson, S. J., Robinson, T. N., Haydel, K. F., y Killen, J. D. (2000). Are overweight children unhappy?: Body mass index, depressive symptoms, and overweight concerns in elementary school children. Archives of Pediatrics y Adolescent Medicine, 154(9), 931-935. https://doi.org/10.1001/archpedi.154.9.931

Estevan, M.C. (2004). Condicionantes Socioeconómicos De Los Hábitos Alimentarios e Ingesta De Energía y Nutrientes En Escolares De La Población Española.

Para citar este artículo utilice la siguiente referencia: Salvador, F.; Muros, J.J.; Gámiz, V.Ma.; Zurita, F. (2021). Revisión teórica de programas de educación para la salud en España. Sportis Sci J, 7 (1), 1-24. https://doi.org/10.17979/sportis.2021.7.1.6530 


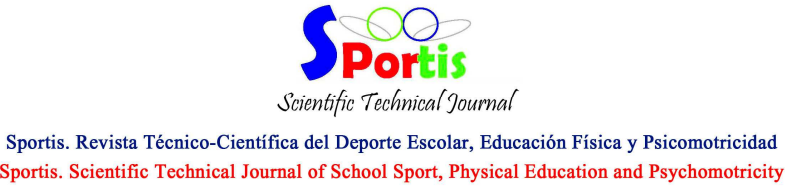

Artículo Original. Revisión teórica de programas de educación para la salud en España. Vol. 7, n. ${ }^{\circ}$ 1; p. 1-24, enero 2021. A Coruña. España ISSN 2386-8333

Evaristo, O.S., Moreira, C., Lopes, L., Abreu, S., Agostinis-Sobrinho, C., Oliveira-Santos J, et al. (2018). Associations between physical fitness and adherence to the Mediterranean diet with health-related quality of life in adolescents: results from the LabMed Physical Activity Study. Eur J Public Health, 28(4), 631-635. https://doi.org/10.1093/eurpub/cky043

Fernández-Morales, I., Aguilar-Vilas, M., Mateos-Vega, C. J., y Martínez-Para, M. (2008). Relación entre la calidad del desayuno y el rendimiento académico en adolescentes de Guadalajara (Castilla-La Mancha). Nutrición Hospitalaria, 23(4), 383-387.

Fraile, A., y De Diego, R. (2006). Motivaciones de los escolares europeos para la práctica del deporte escolar. Un estudio realizado en España, Italia, Francia y Portugal. Revista Internacional De Sociología, 64(44), 85-109. https://doi.org/10.3989/ris.2006.i44.29

French, S. A., Story, M., y Perry, C. L. (1995). Self-esteem and obesity in children and adolescents: A literature review. Obesity Research, 3(5), 479-490. https://doi.org/10.1002/j.1550-8528.1995.tb00179.x

García, F., Míguez, M., y De la Montaña, J. (2011). Relación entre el nivel de actividad física en escolares durante el fin de semana y la prevalencia de obesidad. CyTA - Journal of Food, 9, 295-300. https://doi.org/10.1080/19476337.2011.601431

García-Hermoso, A., Escalante, Y., Domínguez, A. M., y Saavedra, J. M. (2013). Efectos de un programa de ejercicio físico durante tres años en niños obesos: un estudio de intervención. Retos. Nuevas tendencias en Educación Física, Deporte y Recreación, 23, 10-13. https://doi.org/10.20868/upm.thesis.39683

Gidding, S. S., Bao, W., Srinivasan, S. R., y Berenson, G. S. (1995). Effects of secular trends in obesity on coronary risk factors in children: The bogalusa heart study. The Journal of Pediatrics, 127(6), 868-874. https://doi.org/10.1016/s0022-3476(95)70020-x

Gómez, B. V. (2001). La cultura física y las diferencias de género en el umbral del siglo XXI. En La educación física, el deporte y la salud en el siglo XXI (pp. 213-228). Marfil. https://doi.org/10.35376/10324/16240

González, J.M., Sarría, L. y Coca, S. (2004). Cinco años, una vida. Actividad física y hábitos sanos en la adolescencia. Donostia-San Sebastián: Erein.

González-Jiménez, E., Cañadas, G. R., Lastra-Caro, A., y Cañadas-De la Fuente, G. A. (2014). Efectividad de una intervención educativa sobre nutrición y actividad física en una población de adolescentes. Prevención de factores de riesgos endocrinometabólicos y cardiovasculares. Aquichan, 14(4), 549-559. https://doi.org/10.5294/aqui.2014.14.4.9

González-Pascual, M., Manrique-Arribas, J. C., y López-Pastor, V. M. (2012). Valoración del primer curso de implantación de un programa municipal integral de deporte escolar. Retos. Nuevas tendencias en Educación Física, Deporte y Recreación, 21, 1418. https://doi.org/10.35376/10324/1025

Para citar este artículo utilice la siguiente referencia: Salvador, F.; Muros, J.J.; Gámiz, V.Må.; Zurita, F. (2021). Revisión teórica de programas de educación para la salud en España. Sportis Sci J, 7 (1), 1-24. https://doi.org/10.17979/sportis.2021.7.1.6530 http://revistas.udc.es/ 


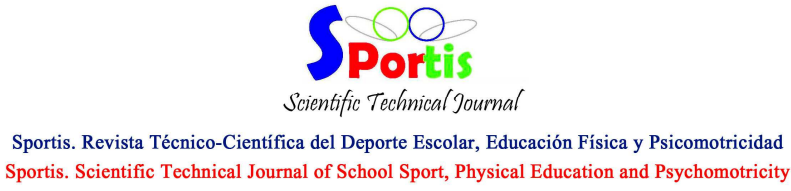

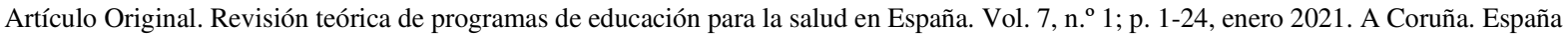
ISSN 2386-8333

Gortmaker, S. L., Must, A., Cobol, A. M., Peterson, K., Cowlitz, G. A., y Dietz, W. H. (1996). Television viewing as a cause of increasing obesity among children in the United States, 1986-1990. Archives of Pediatrics y Adolescent Medicine, 150(4), 356362. https://doi.org/10.1001/archpedi.1996.02170290022003

Granda, J., Alemany, I., y Cortijo, A. (2016). Physical self-concept in primary education. A proposal for a measurement tool. European Journal of Human Movement, 37, 122142.

Granda, J., Montilla, M., Barbero, J. C., Mingorance, Á., y Alemany, I. (2010). Frecuencia de práctica y motivos de participación/no participación en actividades físicas en función del género de escolares de 10-12 años de Melilla. RICYDE. Revista Internacional de Ciencias del Deporte, 6(21), 280-296. https://doi.org/10.5232/ricyde2010.02103

Gutiérrez-Abad, C., San José, M. N., Castroviejo, M., Sánchez, R., y Cordero, J. A. (2015). Enuresis nocturna. Estudio epidemiológico en población escolar. Revista de Pediatría en Atención Primaria, 17, 227-234. https://doi.org/10.4321/s113976322015000400004

Iturbe Lete, A., Emparanza Knör, J., y Perales Antón, A. (1999). Modelo dietético de los adolescentes en Guipúzcoa. Anales Españoles De Pediatría, 50(5), 471-478.

Iturbe-Lete, A., y Perales-Antón, A. (2002). Evaluación del programa de intervención dietética (escolares de $3^{\circ}$ ESO). Nutrición Hospitalaria, 17(6), 296-301.

Lafuente, P. J., Gómez-Pérez de Mendiola, F. J., Aguirre, B., Zabala-Galán, J., IrurzunZuazabal, E., y Gorritxo-Gil, B. (2002). Estilos de vida determinantes de la salud oral en adolescentes de Vitoria-Gasteiz: evaluación. Atención Primaria, 29(4), 213-217. https://doi.org/10.1016/s0212-6567(02)70546-5

Laguna, M., Lara, M ${ }^{a}$ T. y Aznar, S. (2011). Patrones de Actividad Física en función del género y los niveles de obesidad en población infantil española. Estudio EYHS. Revista de Psicología del Deporte, $20 \quad$ (2), 621-636. https://doi.org/10.15366/rimcafd2018.72.002

Llauradó, E., Tarro, L., Moriña, D., Queral, R., Giralt, M., y Solà, R. (2014). EdAl-2 (Educacio en Alimentacio) programme: reproducibility of a cluster randomised, interventional, primary-school-based study to induce healthier lifestyle activities in children. BMJ open, 4(11), e005496. https://doi.org/10.1136/bmjopen-2014-005496

Lois-Carro, L., y Rial-Rebullido, T. (2016). Hábitos alimentarios y de actividad física de alumnado de Educación Primaria: estudio descriptivo de un colegio de Pontevedra. Sportis, 2(1), 77-92. https://doi.org/10.17979/sportis.2016.2.1.1442

López-Sánchez, G., López, J., y Díaz-Suárez, A. (2016). Efectos de un programa de actividad física intensa en la composición corporal de adolescentes murcianos. SPORT TKRevista EuroAmericana de Ciencias del Deporte, 5(2), 83-88. https://doi.org/10.6018/264711

Para citar este artículo utilice la siguiente referencia: Salvador, F.; Muros, J.J.; Gámiz, V.Må.; Zurita, F. (2021). Revisión teórica de programas de educación para la salud en España. Sportis Sci J, 7 (1), 1-24. https://doi.org/10.17979/sportis.2021.7.1.6530

http://revistas.udc.es/ 


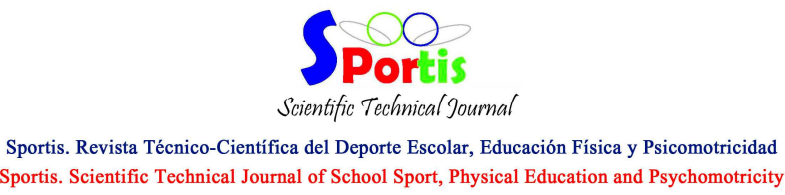

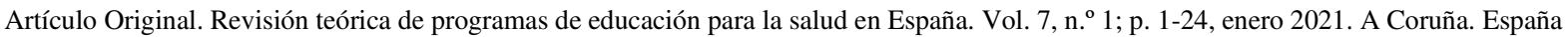
ISSN 2386-8333

Macedo, E., Valverde, M., López, R., Rodríguez, A., López, C., Delgado, C., ... et al. (2007). Dirección General de Promoción de la Salud (Ed.), Manual. Cómo mejorar en la escuela la alimentación de niñas, niños y las y los adolescentes. México. https://doi.org/10.15220/978-958-8514-41-3-sp

Macías, A. I., Gordillo, L. G., y Camacho, E. J. (2012). Hábitos alimentarios de niños en edad escolar y el papel de la educación para la salud. Revista Chilena De Nutrición, 39(3), 40-43. https://doi.org/10.4067/s0717-75182012000300006

Mariscal-Arcas, M., Rivas, A., Velasco, J., Ortega, M., Caballero, A. M., y Olea-Serrano, F. (2009). Evaluation of the Mediterranean diet quality index (KIDMED) in children and adolescents in southern Spain. Public Health Nutrition, 12(09), 1408-1412. https://doi.org/10.1017/s1368980008004126

Martín, M., Tercedor, P., Pérez, I., Chillón, P., y Delgado, M. (2004). Los adolescentes españoles ante la práctica de actividad física y deporte. Estudio Avena. III Congreso Vasco Del Deporte. Deporte y Socialización, Vitoria-Gasteiz. https://doi.org/10.4321/s1578-84232012000100005

Martínez, M. I, Hernández, M. D., Ojeda, M., Mena, R., Alegre, A., y Alfonso, J. L. (2009). Desarrollo de un programa de educación nutricional y valoración del cambio de hábitos alimentarios saludables en una población de estudiantes de Enseñanza Secundaria Obligatoria. Nutrición Hospitalaria, 24(4), 504-510. https://doi.org/10.20960/nh.391

Martínez-García, A., y Trescastro-López, E. M. (2016). Actividades de educación alimentaria y nutricional en escolares de $3^{\circ}$ de primaria en el Colegio Público" La Serranica" de Aspe (Alicante): experiencia piloto. Revista Española de Nutrición Humana y Dietética, 20(2), 97-103. https://doi.org/10.14306/renhyd.20.2.192

Martínez-Martínez, J., Contreras, O. R., Aznar, S., y Lera, Á. (2012). Niveles de actividad física medido con acelerómetro en alumnos de $3^{\circ}$ ciclo de Educación Primaria: actividad física diaria y sesiones de Educación Física. Revista de Psicología del Deporte, 21(1), 117-123. https://doi.org/10.24310/riccafd.2020.v9i2.7155

Martínez-Vizcaíno, V., Aguilar, F. S., Gutiérrez, R. F., Martínez, M. S., López, M. S., Martínez, S. S., ... y Artalejo, F. R. (2008). Assessment of an after-school physical activity program to prevent obesity among 9-to 10-year-old children: a cluster randomized trial. International Journal of Obesity,32(1), 12. https://doi.org/10.1038/sj.ijo.0803738

Martín-Martínez I., Chirosa-Ríos L., Reigal-Garrido R., Hernández-Mendo A., Juárez-Ruizde-Mier R. y Guisado-Barrilao R. (2015). Efectos de la actividad física sobre las funciones ejecutivas en una muestra de adolescentes. Anales de Psicología, 31(3) 962971. https://doi.org/10.6018/analesps.31.3.171601

Para citar este artículo utilice la siguiente referencia: Salvador, F.; Muros, J.J.; Gámiz, V.Mª.; Zurita, F. (2021). Revisión teórica de programas de educación para la salud en España. Sportis Sci J, 7 (1), 1-24. https://doi.org/10.17979/sportis.2021.7.1.6530 http://revistas.udc.es/ 

ISSN 2386-8333

Molinero, O., Castro-Piñero, J., Ruiz, J. R., González Montesinos, J. L., Mora, J., y Márquez, S. (2010). Conductas de salud en escolares de la provincia de Cádiz. Nutrición Hospitalaria, 25(2), 280-289.

Morán, L. J., y Rivera, A. (2015). Alimentación en comedores escolares. Nutrición Clínica en Medicina, 9(3), 204-218.

Morenilla, L. y Iglesias, E. (2012). Relación entre hábitos de práctica deportiva y condición física en adolescentes de Galicia. Apunts Educación Física y Deportes, 107 (1), 24-34. https://doi.org/10.5672/apunts.2014-0983.es.(2012/1).107.02

Moreno, J. A., Cervelló, E., y Borges, F. (2010). 20 estrategias para aumentar la motivación en programas de ejercicio físico. Sevilla: Wanceulen.

Moreno, J. Galiano, M. J. (2006). La comida en familia: Algo más que comer juntos. Acta Pediátr Española, 64 (554), 8.

Moreno, L., Sarria, A., y Popkin, B. (2002). ORIGINAL COMMUNICATION the nutrition transition in Spain: A European Mediterranean country. European Journal of Clinical Nutrition, 56, 992-1003. https://doi.org/10.1038/sj.ejcn.1601414

Moysés, S. J., Moysés, S. T., y Krempel, M. C. (2004). Avaliando o processo de construção de políticas públicas de promoção de saúde: A experiência de curitiba. Ciênc Saúde Coletiva, 9(3), 627-641. https://doi.org/10.1590/s1413-81232004000300015

Muñoz-Heras, A., Fernández, N., y Navarro, R. (2015). Estudio descriptivo sobre los hábitos saludables en alumnado de Primaria desde la educación física escolar. Sportis, 1(1), 87-104. https://doi.org/10.17979/sportis.2015.1.1.1402

Muros, J. J., Zabala, M., Oliveras-López, M. J., Ocaña-Lara, F. A., y de la Serra, H. L. G. (2013). Results of a 7-week school-based physical activity and nutrition pilot program on health-related parameters in primary school children in southern Spain. Pediatric exercise science, 25(2), 248-261. https://doi.org/10.1123/pes.25.2.248

Navarro-Solera, M., González-Carrascosa, R., y Soriano, J. M. (2014). Estudio del estado nutricional de estudiantes de educación primaria y secundaria de la provincia de valencia y su relación con la adherencia a la dieta mediterránea. Revista Española De Nutrición Humana y Dietética, 18(2), 81-88. https://doi.org/10.14306/renhyd.18.2.65

Nieto, F. J., Szklo, M., y Comstock, G. W. (1992). Childhood weight and growth rate as predictors of adult mortality. American Journal of Epidemiology, 136(2), 201-213. https://doi.org/10.1093/oxfordjournals.aje.a116486

Nieto-García, V. M., Nieto-García, M. A., Lacalle-Remigio, R. y Abdel-Kader Martín, L. (2001). Salud oral de los escolares de Ceuta: influencias de la edad, el género, la etnia y el nivel socioeconómico. Revista Española de Salud Pública, 75(6), 541-550. https://doi.org/10.1590/s1135-57272001000600006

Oliva Rodríguez, R., Tous Romero, M., Gil Barcenilla, B., Longo Abril, G., Pereira Cunill, J. L., y García Luna, P. P. (2013). Impacto de una intervención educativa breve a

Para citar este artículo utilice la siguiente referencia: Salvador, F.; Muros, J.J.; Gámiz, V.Ma.; Zurita, F. (2021). Revisión teórica de programas de educación para la salud en España. Sportis Sci J, 7 (1), 1-24. https://doi.org/10.17979/sportis.2021.7.1.6530

http://revistas.udc.es/ 


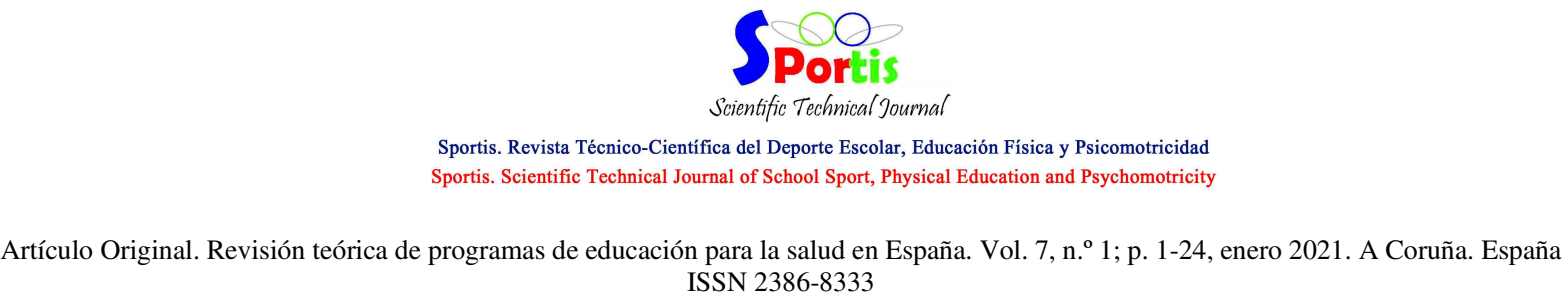

escolares sobre nutrición y hábitos saludables impartida por un profesional sanitario. Nutrición Hospitalaria, 28(5), 1567-1573.

Ortiz-Andrellucchi, A., Peña Quintana, L., Albino Beñacar, A., Mönckeberg Barros, F., y Serra-Majem, L. (2006). Desnutrición infantil, salud y pobreza: Intervención desde un programa integral. Nutrición Hospitalaria, 21(4), 533-541. https://doi.org/10.1111/j.1753-4887.2009.00159.x

Oviedo, G., Sánchez, J., Castro, R., Calvo, M., Sevilla, J. C., Iglesias, A., y Guerra, M. (2013). Niveles de actividad física en población adolescente: estudio de caso. Retos. Nuevas tendencias en Educación Física, Deporte y Recreación, 23, 43-47. https://doi.org/10.24054/16927427.v1.n1.2017.3355

Pearson, N., y Biddle, S. J. (2011). Sedentary behavior and dietary intake in children, adolescents, and adults: A systematic review. American Journal of Preventive Medicine, 41(2), 178-188. https://doi.org/10.1016/j.amepre.2011.05.002

Pérez Gallardo, L., Bayona, I., Mingo, T., y Rubiales, C. (2011). Utilidad de los programas de educación nutricional para prevenir la obesidad infantil a través de un estudio piloto en Soria. Nutrición Hospitalaria, 26(5), 1161-1167.

Pérez-Solís, D., Díaz-Martín, J.J., Álvarez-Caro, F., Suárez-Tomás, I., Suárez-Menéndez, E., y Riaño-Galán, I. (2015). Efectividad de una intervención escolar contra la obesidad. Anales de Pediatría, 83(1) 19-25. https://doi.org/10.1016/j.anpedi.2014.08.010

Petry, N. M., Barry, D., Pietrzak, R. H., y Wagner, J. A. (2008). Overweight and obesity are associated with psychiatric disorders: Results from the national epidemiologic survey on alcohol and related conditions. Psychosomatic Medicine, 70(3), 288-297. https://doi.org/10.1097/psy.0b013e3181651651

Pin-Arboledas, G., Alarcón, M. C., González, G. M., Roselló, A. L., y Salort, M. M. (2011). Hábitos y problemas con el sueño de los 6 a los 14 años en la Comunidad Valenciana. opinión de los propios niños. Anales de Pediatría, 74(2), 103-115. https://doi.org/10.1016/j.anpedi.2010.08.014

Poletti, C., Oscar, H., y Lilian Barrios, M. (2003). Sobrepeso y obesidad como componentes de la malnutrición, en escolares de la ciudad de corrientes: Argentina. Revista Chilena De Pediatría, 74(5), 499-503. https://doi.org/10.4067/s0370-41062003000500006

Puig, M. S., Moñino, M., Colomer, M., Martí, N., y Muñoz, E. (2006). Modificación de los hábitos alimentarios en escolares del término municipal de Llucmajor tras dos años de intervenciones educativas (2002-2005). Revista Española de Nutrición Comunitaria, 3, 14-22. https://doi.org/10.1016/s1135-3074(10)70008-9

Ramos, S. (2013). Desplazamiento activo escolar. Trabajo Fin de Grado: Universidad de Valladolid.

Rey, J., Bresson, J., y Abadie, V. (1994). La nutrition, un modèle d'interaction entre les facteurs génétiques et les facteur d'environnement. Archives De Pédiatrie, 1(1), 510.

Para citar este artículo utilice la siguiente referencia: Salvador, F.; Muros, J.J.; Gámiz, V.Ma.; Zurita, F. (2021). Revisión teórica de programas de educación para la salud en España. Sportis Sci J, 7 (1), 1-24. https://doi.org/10.17979/sportis.2021.7.1.6530

http://revistas.udc.es/ 


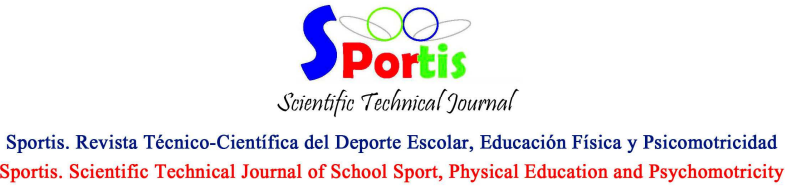

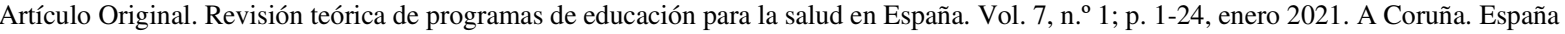
ISSN 2386-8333

Robles, M. I., Obando, J., González, M. T., y Bueno, J. (2011). Asociación entre dieta, actividad física y consumo de tabaco en adolescentes. SEMERGEN-Medicina de Familia, 37(5), 238-245. https://doi.org/10.1016/j.semerg.2011.01.004

Rodríguez, F., Banegas, J., Graciani, M., Hernández Vecino, R., y Rey Calero, J. D. (1996). El consumo de alimentos y nutrientes en España en el periodo 1940-1988. I. análisis de su consistencia con la dieta mediterránea. Medicina Clínica, 106(5), 161-168. https://doi.org/10.1016/s0025-7753(01)71866-0

Ruiz-Santana, S., Ruiz, F., Hernández, C. R., Álvarez, A., y Saavedra, P. (2009). Evaluación de dos intervenciones nutricionales y de hábitos de higiene y salud en una cohorte de alumnos de enseñanza primaria. Nutrición Hospitalaria, 24(5), 596-606.

Samuelson, G., Bratteby, L., Enghardt, H., y Hedgren, M. (1996). Food habits and energy and nutrient intake in Swedish adolescents approaching the year 2000. Acta Pediátrica, 85(s415), 1-19. https://doi.org/10.1111/j.1651-2227.1996.tb14268.x

Sánchez-Rivas, E., Mayorga-Vega, D., Fernández-Rodríguez, E. y Merino-Marbán, R. (2014). Efecto de un programa de estiramiento de la musculatura isquiosural en las clases de educación física en Educación Primaria. Journal of Sport and Health Research, 6 (2), 159-168. https://doi.org/10.24310/riccafd.2017.v6i1.3803

Sánchez-Villegas, A., Bes-Rastrollo, M., Martínez-González, M., y Serra-Majem, L. (2006). Adherence to a Mediterranean dietary pattern and weight gain in a followup study: The SUN cohort. International Journal of Obesity, 30(2), 350-358. https://doi.org/10.1038/sj.ijo.0803118

Sancho-González, L., Pérez-Patrón, G., Torres-Asensio, M., y Campillo-Álvarez, J. (2002). Estilo de vida y hábitos alimentarios de los adolescentes extremeños. SEMERGENMedicina de Familia, 28(4), 177-184. https://doi.org/10.1016/s1138-3593(02)74052-5

Serdula, M. K., Ivery, D., Coates, R. J., Freedman, D. S., Williamson, D. F., y Byers, T. (1993). Do obese children become obese adults? A review of the literature. Preventive Medicine, 22(2), 167-177. https://doi.org/10.1006/pmed.1993.1014

Serra-Majem, L., García-Closas, R., Ribas, L., Pérez-Rodrigo, C., y Aranceta, J. (2001). Food patterns of Spanish schoolchildren and adolescents: The enKid study. Public Health Nutrition, 4(6a), 1433-1438. https://doi.org/10.1079/phn2001234

Serra-Paya, N., Solé, A. E., y Nespereira, A. B. (2014). Intervención multidisciplinar y no competitiva en el ámbito de la salud pública para el tratamiento del sedentarismo, el sobrepeso y la obesidad infantil: Programa NEREU. Apunts. Educación física y deportes, 3(117), 7-22. https://doi.org/10.5672/apunts.2014-0983.es.(2014/3).117.01

Speiser, P. W., Rudolf, M. C., Anhalt, H., Camacho-Hubner, C., Chiarelli, F., Eliakim, A., ... Iughetti, L. (2005). Childhood obesity. The Journal of Clinical Endocrinology $y$ Metabolism, 90(3), 1871-1887. https://doi.org/10.1210/jc.2004-1389

Sullivan, M., Karlsson, J., Sjostrom, L., Backman, L., Bengtsson, C., Bouchard, C., ... Lindstedt, S. (1993). Swedish obese subjects (SOS)--an intervention study of obesity. Para citar este artículo utilice la siguiente referencia: Salvador, F.; Muros, J.J.; Gámiz, V.Ma .; Zurita, F. (2021). Revisión teórica de programas de educación para la salud en España. Sportis Sci J, 7 (1), 1-24. https://doi.org/10.17979/sportis.2021.7.1.6530 http://revistas.udc.es/ 


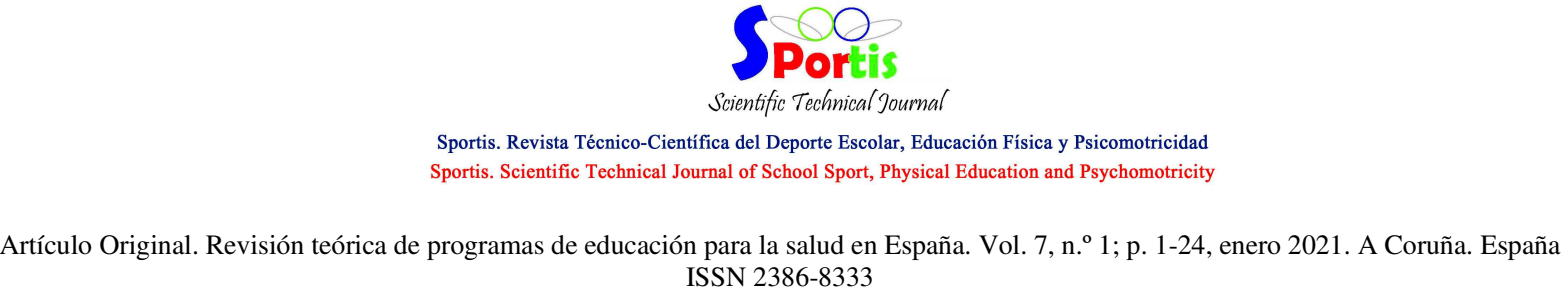

Baseline evaluation of health and psychosocial functioning in the first 1743 subjects examined. International Journal of Obesity and Related Metabolic Disorders : Journal of the International Association for the Study of Obesity, 17(9), 503-512. https://doi.org/10.1002/0470846739.ch35

Tercedor, P., Martín-Matillas, M., Chillón, P., Pérez López, I. J., Ortega, F. B., Wärnberg, J., ... y Delgado, M. (2007). Incremento del consumo de tabaco y disminución del nivel de práctica de actividad física en adolescentes españoles: Estudio AVENA. Nutrición Hospitalaria, 22(1), 89-94. https://doi.org/10.4321/s1578-84232012000100005

Tobisch, B., Blatniczky, L., y Barkai, L. (2015). Cardiometabolic risk factors and insulin resistance in obese children and adolescents: Relation to puberty. Pediatric Obesity, 10(1), 37-44. https://doi.org/10.1111/j.2047-6310.2013.00202.x

Tojo, R., y Leis, R. (2007). Alimentación del niño escolar. Manual Práctico De Nutrición Pediatría. Ergon, Madrid.

Torres-Luque, G., Carpio, E., Lara, A. y Zagalaz, M. L. (2014). Niveles de condición física de escolares de educación primaria en relación a su nivel de actividad física y al género. Retos. Nuevas tendencias en Educación Física, Deporte y Recreación, 25, 17-22. https://doi.org/10.24310/riccafd.2020.v9i2.7155

Tur, J. A., Romaguera, D., y Pons, A. (2004). Food consumption patterns in a Mediterranean region: Does the Mediterranean diet still exist? Annals of Nutrition y Metabolism, 48(3), 193-201. https://doi.org/10.1159/000079754

Tur, J. A., Serra-Majem, L., Romaguera, D., y Pons, A. (2005). Does the diet of the balearic population, a Mediterranean type diet, still provide adequate antioxidant nutrient intakes? European Journal of Nutrition, 44(4), 204-213. https://doi.org/10.1007/s00394-004-0512-0

Urrutia-Rojas, X., Egbuchunam, C. U., Bae, S., Menchaca, J., Bayona, M., Rivers, P. A., y Singh, K. P. (2006). High blood pressure in school children: Prevalence and risk factors. BMC Pediatrics, 6, 32. https://doi.org/10.1186/1471-2431-6-32

Vareiro, D., Bach-Faig, A., Raidó Quintana, B., Bertomeu, I., Buckland, G., Vaz de Almeida, María Daniel, y Serra-Majem, L. (2009). Availability of Mediterranean and nonMediterranean foods during the last four decades: Comparison of several geographical areas. Public Health Nutrition, 12(9A), 1667-1675. https://doi.org/10.1017/s136898000999053x

Vázquez, B., Fernández, E., Ferro, S., Learreta, B., y Viejo, J. (2000). Educación física y género. Madrid, Gymnos.

Zamacona, N., Rocandio Pablo, A. M., Municio, M., Ansotegui Alday, L., Martínez de la Pera, C., y Arroyo Izaga, M. (2011). Consumo de alimentos ricos en azúcar-sal en niños de Las Arenas (Getxo, Bizkaia) por grupos de edad. Zainak. 34, 311-321.

Para citar este artículo utilice la siguiente referencia: Salvador, F.; Muros, J.J.; Gámiz, V.Ma.; Zurita, F. (2021). Revisión teórica de programas de educación para la salud en España. Sportis Sci J, 7 (1), 1-24. https://doi.org/10.17979/sportis.2021.7.1.6530

http://revistas.udc.es/ 